\title{
Why We Need Continuous Pharmaceutical Manufacturing and How to Make it Happen
}

A Summary of Thoughts from the International Symposium on Continuous Manufacturing

Clive Badman, Professor of Practice at University of Strathclyde

Charles L Cooney, Robert T. Haslam (1911) Professor of Chemical Engineering, Emeritus, MIT

Alastair Florence, Professor and Director, EPSRC Future Continuous Manufacturing and

Advanced Crystallisation Hub (CMAC))

Konstantin Konstantinov, Executive Vice-President Manufacturing Sciences, Codiak

BioSciences

Markus Krumme, Head, Continuous Unit, Novartis Pharmaceuticals, Inc.

Salvatore Mascia, CONTINUUS Pharmaceuticals, Inc.

Moheb Nasr, Nasr Pharma Regulatory Consulting (NPRC)

Bernhardt L. Trout, Raymond F. Baddour, ScD, (1949) Professor of Chemical Engineering, MIT

\section{Where We Are}

Most industries as they evolve to meet market needs have embraced continuous manufacturing, a combination of continuous movement of components, a systems approach to designing processes and automated model-based control. Such an approach has led to tremendous increases in efficiency and quality. Among major industries, the exception is the pharmaceutical and biotech industry. Despite a long time acknowledgement of the benefits of continuous manufacturing in this industry, adoption has been slow and piecemeal. In 2007, after many years discussing continuous manufacturing in industry and academics, Dr. Janet Woodcock, CDER Director, USFDA, at the inauguration of the Novartis-MIT Center for Continuous Manufacturing challenged the industry to accelerate its adoption. In 2011, the first end-to-end continuous pharmaceutical process with automated, model-based control was run at MIT (bench scale). Thereafter followed three International Symposia on Continuous Manufacturing of Pharmaceuticals (ISCMP) in 2014, 2016 and 2018, held in the US and UK. The outputs have included highly cited white papers in this journal on continuous pharmaceutical technology, supply chain and regulatory approaches. There are now six drugs, all small-molecule pharmaceuticals, that incorporate continuous manufacturing, but only partially and with incorporation of basic technologies. Vertex's Orkambi and Symdeko, Johnson \& Johnson's Prezista, Eli Lilly's Verzenio and Pfizer's Daurismo have been approved in the US; Vertex's Orkambi and Symkevi, Johnson \& Johnson's Prezista, and Eli Lilly's Verzenio in the EU; and Johnson \& Johnson's Trmacet and Eli Lilly's Verzenio in Japan. While the progress is acknowledged by the pharmaceutical community, for 12 years of development it is hardly admirable. The situation with biomanufacturing is similar. While continuous bioprocessing has been successfully implemented commercially for upstream (perfusion cell culture) for more than 25 years, integration of the downstream operation (chromatography and filtration) has not yet been demonstrated commercially. The irony is that none of the approved continuous processes had any major regulatory challenges related to the technologies, including control, and regulators keep encouraging development of new innovative processes, but still progress is slow.

The rate of progress was particularly evident at the 2018 International Symposium on Continuous Manufacturing of Pharmaceuticals, where there was continued enthusiasm for continuous 


\section{J. Pharm. Sci., in press}

manufacturing amongst the technical and quality community, but acknowledgement that the pace of implementation is slower than anticipated. The irony is that amongst this same community, there is industry-wide agreement that continuous pharmaceutical manufacturing would provide tremendous benefits, including substantial cost benefits, and regulators do not see scientific barriers per se and find the successes to date encouraging. However, the industry, like a car with its wheels stuck in mud, seems unable to get out of its rut. If only we could get on the road of continuous manufacturing, we would be cruising ahead, but as for now, we are spinning our wheels, inching forward.

After the 2018 symposium, over a decade after Dr. Woodcock's historic speech, we intended to provide a summary of the progress in continuous manufacturing. However, since it is evident that progress is slow, we decided to act more directly to try to pull the continuous manufacturing vehicle out of the mud. Here we provide a summary of the reasons why we believe the industry is stuck and how to get it unstuck. It has become evident that major policy initiatives are needed.

\section{A Case for Continuous Manufacturing}

The pharmaceutical and biotech industry has unprecedented impact on health care, providing to patients therapeutic solutions to unmet medical needs and broadening patient access to medicine. Investment in life science research continues to identify innovative therapeutic solutions to life threatening diseases, increasing the quality of patient's lives. However, much remains to be done to assure our goal of sustained delivery of quality healthcare to more patients. Even as innovative new therapeutic approaches help more and more patients and lead to more personalized medicine, manufacturing tends to be slow to innovate. Yet manufacturing plays an essential, enabling role as it lies on the critical path to deliver the benefits of medical science to patients. Similarly, for established therapeutics, the potential of manufacturing innovation is far from achieved, and yet would provide tremendous benefits. Historically, in most every industry, in order to meet demand for quality and access, manufacturing has moved through a strategy of intensification and integration to continuous manufacturing. While the pharmaceutical and biotech industry has pursued such a strategy, and we see manufacturing innovation, the pace is slow.

The metrics of progress in addressing our goal are: speed, value (with cost being an element), flexibility, and quality. Assurance of quality through the guaranteed provision of a safe and efficacious medicine is sacrosanct; speed from discovery to the patient is critical; cost across the discovery-to-patient lifecycle is important to the innovator, payer and patient; similarly, cost and quality are key post-patent for generic and biosimilar manufacturers; and flexibility to meet dynamic market demands across the life-cycle and beyond is essential to assure sustained access. The case for accelerating the pace of manufacturing innovation should be made through understanding the needs of the patient, the business and the regulator.

- The patient need for access to medicine for acute and chronic care is clear; improved care is essential to enhanced quality of life and economic prosperity, in particular when faced with an ageing population. The pace of scientific discovery creates an urgency to translate these benefits promptly to the patient. Established medicines need to be available to meet demand. 
- The industry plays a critical role as translator of scientific discovery through a journey of drug development, clinical evaluation and manufacturing that enables patient's access to medicine. The translation is both uncertain and expensive across a lengthy timeline to assure safe and efficacious products for the patient. Supply chain security is critical. The business case for investment is essential; the development time to reach the patient and cost are critical. Given the market uncertainty that depends on clinical efficacy, competitive therapeutic strategies and payer capabilities, manufacturing flexibility across a product's lifecycle is very important. The situation is analogous for established medicines, where risks in a highly regulated environment must correspond to potential rewards.

- The regulator has a responsibility to the patient and to society to assure the sustained availability of quality products to the patient.

Progress to date in implementing continuous manufacturing through process intensification and integration is promising. Measurement of drug critical quality attributes demonstrate a reduction in variance. Manufacturing cycle time through integrated process steps are dramatically shorter, contributing to reduced costs and increased flexibility. Process equipment size compared to batch is smaller, and continuous processes often incorporate fewer steps resulting in lower capital and operating cost and permitting greater flexibility.

Given this, the tangible benefits of continuous manufacturing include:

- Much faster and more economic transition from small scale clinical manufacturing to launch scale.

- High potential for enhanced domestic manufacturing, which includes supply-chain and security benefits. This is because unlike batch processes, continuous processes do not depend on low-cost labor, but instead on advanced technology.

- Improved national security by reducing dependency on foreign suppliers, with consequent higher quality assurance of products manufactured within the country.

- Reduction of stockpiles for necessary medicines, which are discarded upon expiration, as more and more pharmaceuticals could be produced on demand. This would have a major impact on medical countermeasure strategy.

- Potential for a significant improvement in process quality and consistency due to the unprecedented ability to maintain a state of control, low residence times, and no intermediate hold steps.

- Ability to respond much more agilely to drug shortages and related challenges including decreasing the risk of having sole suppliers for essential medicines.

- Lower risk of stock-outs.

- An economically viable route for personalized medicine, of which citizens will want more and more.

- Related to the previous benefit, demand will also increase for new and more complex types of pharmaceuticals like nucleotides, cell and gene therapy, vaccines and advanced biologic modalities.

- Enhanced ability of generic competition as regulatory and economic barriers get lower.

\section{Why we are Stuck}




\section{J. Pharm. Sci., in press}

For the most part, manufacturing innovation and the development of continuous processes and implementation is expensive. The value generation, including cost savings and other advantages as discussed above can be significant in a proper implementation that allows the value it offers to be realized. The challenge arises for a specific pharmaceutical or biotech product in justifying the transition from a known and installed (possibly fully depreciated) base technology with established business and regulatory processes (in multiple countries) at a given company to a new paradigm. There are limitations with availability of skilled personnel and existing large batch manufacturing capacity supporting the current operating model. Because of the significant investment needed, approval for a transition must be made at very senior levels in companies, including the Board. This means there must be significant corporate level support. However, investing in innovative technologies like continuous manufacturing along with new facilities is not a priority in the current business and regulatory environment. As medicines move to a smaller volume paradigm, the business case for major investments is challenging. If the whole industry could move to continuous manufacturing, there would be tremendous value as described above. However, the barriers are currently too high, particularly in light of the shifting paradigm to smaller product volumes. In addition, smaller companies that do not have existing manufacturing assets, are looking to Contract Manufacturing Organizations (CMO's) with continuous manufacturing capabilities, but these are still lacking for the same reasons that major companies have been slow to adopt continuous manufacturing capabilities.

The bottom line is that substantial benefits of continuous manufacturing remain untapped.

\section{How to make it Happen}

Given the need for companies to make major investments to harness the value of continuous manufacturing, we need game-changing incentives for the business as a whole. Traditionally, what has led to industry wide-changes are tax and regulatory incentives that affect cost and time to or in the market. The former have led to Ireland, Singapore and Puerto Rico as key pharmaceutical manufacturing hubs, and the latter include "Breakthrough Therapy" designation and additional patent exclusivity for pediatric indications.

Other on-going activities should be supported as well but are no substitute for these gamechangers. Harmonization of regulatory processes for continuous manufacturing will avoid the costly steps of supplying different markets by two methods, batch and continuous, and would facilitate adoption. Steps are indeed being made, for example with ICH Q13, but these are still several years away. Acceleration of global harmonization would be beneficial. Global approval of manufacturing processes would be most helpful along with having a single world-wide process for regulatory approval of manufacturing changes. Pre-competitive $R \& D$ activities are taking place and are creating opportunities, which can be seized by companies given incentives. Several nations support competitive and collaborative R\&D programs often using national manufacturing research and innovation clusters with investment in the associated infrastructure and technical service support, routes for knowledge exchange and technology transfer and coordinated support for organization and institutions to develop standards. Support for skills development and training of existing and future workforce remain important. Whilst these are necessary aspects of creating the right environment for successful innovation, they are not sufficient alone and do not lead to the needed corporate-level decisions industry-wide. 
Suffice it to say that the incentives that we have in mind must be for significant innovation that will make the business case for transition to continuous with multiple daisy-chained steps favorable. This innovation must follow the letter and spirit of continuous manufacturing described above: a combination of continuous movement of components, a systems approach to designing processes and automated model-based control.

The kind of major incentives that we think would change the playing field are in two categories:

\section{Tax incentives:}

- Tax incentives for investing in pharmaceutical manufacturing innovation including continuous manufacturing R\&D and production.

- Tax incentives for production of pharmaceutical and biopharmaceuticals via continuous manufacturing for brand companies, generics, and biosimilars.

Regulatory incentives that affect time:

- Expedited approval process for both NDA's and ANDA's using continuous manufacturing (similar to break-through therapy status).

- Expedited approvals for sNDA's, in a way that incentivizes moving an existing process to continuous (often easier than developing a new product continuous process).

- Consideration for exclusivity period for drugs manufactured via continuous.

- System-based regulatory oversight instead of evidence based regulations, e.g. creation of rating certificates for companies based on innovation and product quality enhancements to enable simpler and faster regulatory approval or even waivers of certain regulatory aspects.

In summary, the highly regulated pharmaceutical industry is often conservative in its approach to manufacturing innovation and faces complex challenges in introducing new manufacturing technologies. These include the belief that introducing new manufacturing technologies could cause delays to approval or new burdens for the business. The benefits of adoption of continuous manufacturing are immense, but major investments need to be made with corporate-level approval. In order to accelerate adoption and achieve the goals of quality, speed, cost and flexibility, we believe that the above combination of incentives is needed. Such an approach will help ensure that patients benefit from accelerated time from discovery of improved treatments to positive health outcomes as well as assurances in sustained, reliable, cost effective access to quality medicine. 